\title{
Anomalous Reflection of Acoustic Waves in Air with Metasurfaces at Low Frequency
}

\author{
Huaijun Chen (iD) \\ College of Physics and Electronic Information Engineering, Engineering Research Center of Nanostructure and Functional Materials, \\ Ningxia Normal University, Guyuan 756000, China
}

Correspondence should be addressed to Huaijun Chen; chenhuaijun79@163.com

Received 30 October 2017; Accepted 24 December 2017; Published 31 January 2018

Academic Editor: Yuri Tarasevich

Copyright (c) 2018 Huaijun Chen. This is an open access article distributed under the Creative Commons Attribution License, which permits unrestricted use, distribution, and reproduction in any medium, provided the original work is properly cited.

An acoustic metasurface made of a composite structure of cavity and membrane is proposed and numerically investigated. The target frequency is in the low frequency regime $(570 \mathrm{~Hz})$. The unit cells, which provide precise local phase modulation, are rather thin with thickness in the order around $1 / 5$ of the working wavelength. The numerical simulations show that the designed metasurface can steer the reflected waves at will. By taking the advantage of this metasurface, an ultrathin planar acoustic axicon, acoustic lens, and acoustic nondiffracting Airy beam generator are realized. Our design method provides a new approach for the revolution of future acoustic devices.

\section{Introduction}

The rising acoustic metamaterials, whose structures are on a subwavelength scale, exhibit many novel properties that can not be realized by natural materials in controlling sound waves, such as negative mass density, negative modulus, and double-negative parameters [1-10]. Liu et al. embedded rubber-coated lead spheres into epoxy matrix and first realized a metamaterial with negative effective mass density [1]. Fang et al. designed an array of subwavelength Helmholtz resonators and first realized negative effective modulus [2]. However, these metamaterials are all bulk materials and much larger than the working wavelength, which is to the disadvantage of the miniaturization and integration of acoustic devices. Yang et al. designed a kind of metamaterial based on elastic membrane, which effectively reduced the size of metamaterials [11]. In recent years, a new type of metamaterial, namely, metasurface $[12,13]$, attracted the attention of researchers. Metasurface is an artificial lamellar structure composed of units with subwavelength thickness. By arranging the units in a particular way, the metasurface can modulate the direction of scattered waves at will. With this prominent advantage, the metasurface will lead to the revolution of many traditional devices. In the field of optics, the metasurfaces have developed to realize many remarkable functions, such as light beam steering [14], ultrathin optical skin cloak [15], and holograms [16]. As the wavelengths of sound waves are much larger than that of light wave, the acoustic metasurfaces [17-24] are especially important for acoustic devices. Faure et al. realized an acoustic carpet cloaking by using a metasurface made of graded Helmholtz resonators [18]. Li et al. designed coiled space elements and realized a two-dimensional ultrathin acoustic metamaterial to support the extraordinary acoustic transmission [25]. Zhu et al. designed a subwavelength corrugated surface to control the acoustic wavefront arbitrarily without bandwidth limitation [26]. Esfahlani et al. designed a space-coiled cylindrical unit and generated acoustic vortices based on this passive acoustic metasurface [27]. Zhai et al. [24] and Esfahlani et al. [28], respectively, proposed an acoustic metasurface carpet cloak based on membrane-capped cavities. However, the proposed metasurfaces still face the limits of complicated structures, large dimensions, and high working frequencies. Here, considering these factors, we presented a low frequency $(570 \mathrm{~Hz})$ ultrathin acoustic metasurface by using the composite structure of cavity and membrane. This kind of metasurface is capable of arbitrarily steering the reflected waves. Based on this metasurface, we designed some 


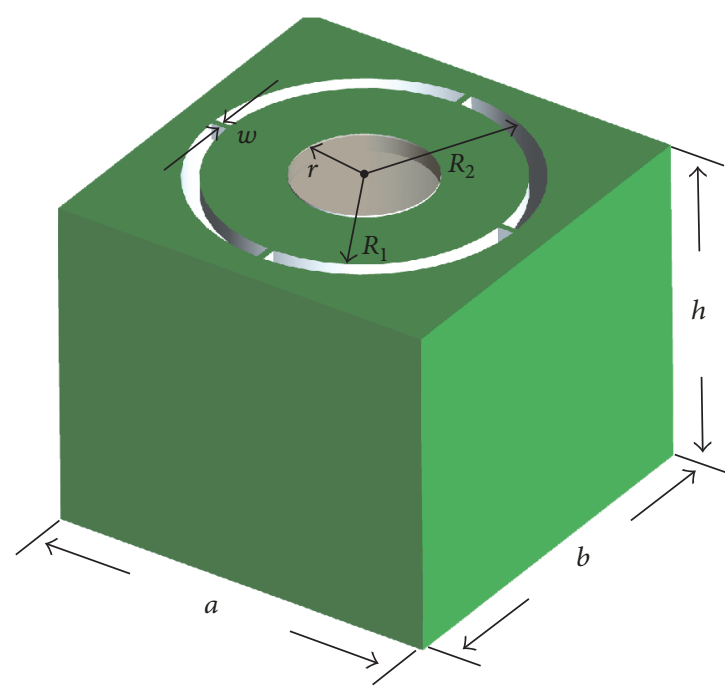

(a)

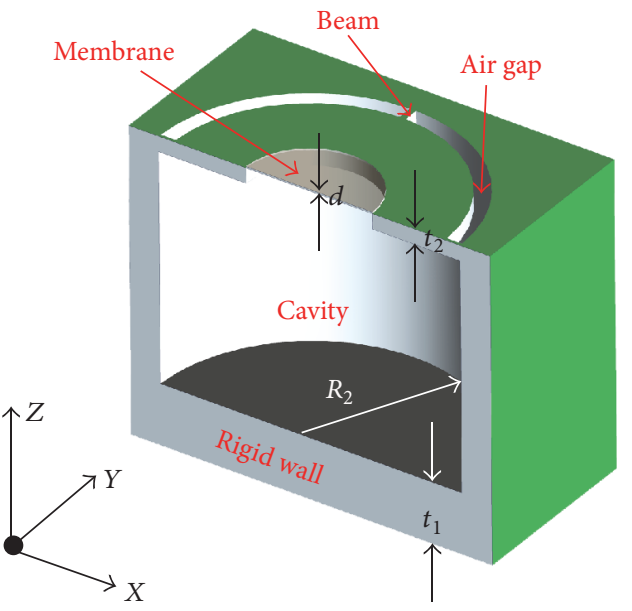

(b)

FIGURE 1: (a) Schematic map of acoustic metasurface unit cell and (b) longitudinal section of the unit cell. The unit cell is composed of a cavity with acoustically hard walls, which is capped with a rigid circular ring. The inside of the ring is a clamped membrane, and the outside is an air gap.

ultrathin acoustic planar devices, such as planar acoustic axicon, acoustic lens, and acoustic nondiffracting Airy beam generator. Our design method provides a new approach for the revolution of future acoustic devices.

\section{Design of the Model}

Figure 1(a) shows the schematic of unit for the planar acoustic metasurface. The unit is a cube (i.e., $a=b=h=$ $120 \mathrm{~mm}$, which is much less than the operating wavelength $\lambda=600 \mathrm{~mm}$ ) with a cylindrical cavity in it. The cavity is filled with air. Figure 1(b) displays the sectional view of the unit. The gray area indicates rigid wall that is opaque to sound waves. The thickness of the bottom wall is $t_{1}=15 \mathrm{~mm}$. The radius $R_{2}$ and height of the cavity are $50 \mathrm{~mm}$ and $100 \mathrm{~mm}$, respectively. An annular lid whose inner circle is sealed by an elastic membrane is put on the top of the cavity. The thickness $t_{2}$ and outer radius $R_{1}$ of the lid are $5 \mathrm{~mm}$ and $45 \mathrm{~mm}$, respectively. The edge of the tensioned membrane is fixed on the side wall. The radius of the membrane is $r$, while the thickness is $d=$ $0.04 \mathrm{~mm}$. There are four beams connecting the lid and the cavity, which produces an annular air gap. The width of the beam is $w=2 \mathrm{~mm}$, which can be ignored comparing to the operating wavelength. The radial width of the gap is $R_{2}-R_{1}$ $=5 \mathrm{~mm}$.

When there is no incident sound wave, the elastic membrane will be tensioned to be a straight line; however, when there are incident waves along the $-z$ direction, the impinging of sound beams on the membrane will lead to forced vibration and deformation of the membrane. At the same time, the compression and expansion of the air in the cavity will result in the air in and out of the cavity through the gap, which greatly reduces the thermal loss of the sound energy. The vibration displacement of the membrane has delay compared with the local velocity of air particles [29]. Compared with the propagation in complete air medium with the same length, there exists substantial phase delay as sound waves travel through the subunit, which shows promise for the altering of reflected phase in space. Thus, the reflected phase of the sound wave will be shifted compared with the incident phase. Previous works have demonstrated that physical parameters of the material (i.e., the mass density, Young's modulus, and Poisson's ratio), the geometric dimensions of the unit, and the working frequency all have influences on the sound behavior of the unit, including reflected ratio and phase [30-32]. In the present study, in order to guarantee the unitary subwavelength thickness of entire metasurface and for the simplicity of design, the thicknesses of the unit and the membrane are fixed. The only variable in this paper is the radius of the membrane $r$. Our following simulations will demonstrate that, by appropriately choosing $r$, eight units are enough to provide discrete reflected phases change ranging from 0 to $2 \pi$ with an interval of $\pi / 4$ between the adjacent units. The commercial software COMSOL 5.2a was employed to calculate the field distribution of reflected sound wave. The chosen medium in the simulation is air. The sound speed and mass density of air are $343 \mathrm{~m} / \mathrm{s}$ and $1.21 \mathrm{~kg} / \mathrm{m}^{3}$, individually. The mass density, Young's modulus, and Poisson's ratio of the membrane are $920 \mathrm{~kg} / \mathrm{m}^{3}, 9.6 \times$ $10^{9} \mathrm{~Pa}$, and 0.36 , individually, so that traditional polyethylene can be suitable for the practical fabrication of this membrane. Based on [30], the damping factor of the membrane material is set to be 0.01 . The side walls were chosen to be aluminum and modeled as acoustically rigid. When we studied the reflected phase of unit with specific membrane radius, the simulation was individually performed for each unit. Under this circumstance, the periodic boundary condition is applied along $x$-axis direction to eliminate the boundary effects. In 


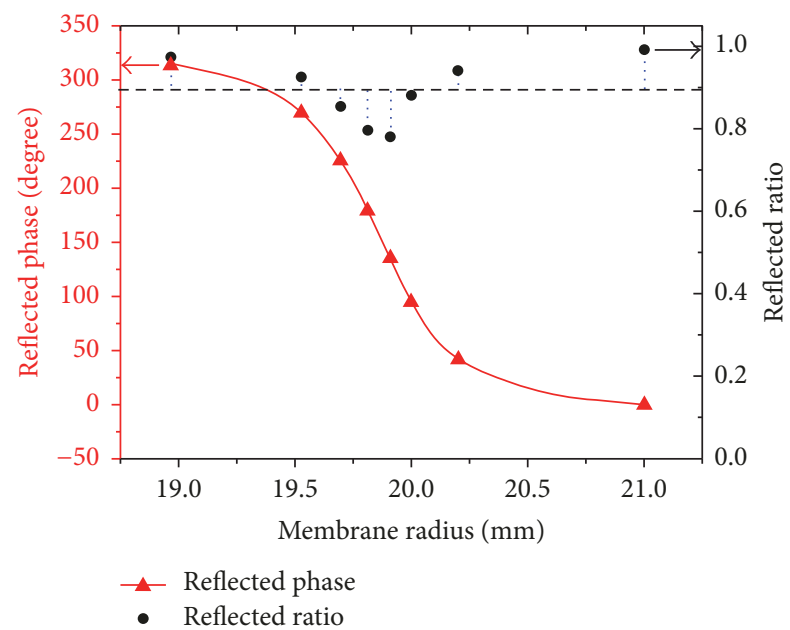

FIGURE 2: Reflected phases and ratios of the eight subunits as a function of $r$. The wavelength $\lambda$ of the incident wave is $600 \mathrm{~mm}$. The red triangles and black dots refer to discrete phases and ratios of eight subunits having specific membrane radius (i.e., 18.97, 19.53, $19.70,19.81,19.91,20.00,20.20$, and $21.00 \mathrm{~mm}$, respectively). The phase shift between two adjacent subunits is $45^{\circ}$. The mean reflected ratio is 0.89 , as shown by the dashed line.

addition, the plane wave radiation boundary condition was applied for the incident plane wave propagating along the negative direction of $z$-axis.

The relation between the simulated reflected phase of the unit and the membrane radius is shown with the red curve in Figure 2. We can see that the designed units are able to realize $2 \pi$ phase change, when the membrane radius varies from $18.97 \mathrm{~mm}$ to $21.00 \mathrm{~mm}$. In this paper, we choose eight units to provide discrete $2 \pi$ phase shifts with an interval of $\pi / 4$ between the adjacent subunits, denoted by the red triangles. In addition, we also obtained the reflected ratios of these eight units, as shown with the black dots in Figure 2. Based on [24], the reflected ratio of the metamaterial with membrane is also low at the resonant frequency, but the reflected ratio will be very high once the frequency is away from the resonant frequency. Therefore, the resonant frequencies of the structural units in this work are not at the designed working frequency (i.e., $570 \mathrm{~Hz}$ ). Thus, it can be guaranteed not to have very low reflectivity, but also to provide sufficient phase delay. The dashed line represents the mean reflected ratio with the value of 0.89 , which demonstrates that these units are suitable for assembling metasurfaces with high efficiency.

\section{Abnormal Reflection of the Metasurface}

The generalized Snell's law (GSL) was introduced to predict the anomalous propagation of incident wave across material interfaces characterized by a phase gradient $[14,20,33,34]$, which is suitable for both reflected wave and transmitted wave. The metasurfaces proposed in this study are also built based on GSL. According to the GSL, the direction of anomalous reflection is related to the direction of the incident planar wave front and the phase gradient at the interface between the metasurface and air. The formula is as follows:

$$
\sin \theta_{r}-\sin \theta_{i}=\frac{1}{k_{0}} \frac{d \phi}{d x}
$$

where $\theta_{i}$ and $\theta_{r}$ are the incident and reflected angles, respectively. $k_{0}=2 \pi / \lambda_{0}$ is the wave vector in air, while $d \phi$ and $d x$ are the phase and distance gradients along the $x$-direction separately. Equation (1) implies that the reflected beam can have an arbitrary direction, provided that a suitable phase gradient is produced along the interface of metasurface. In order to verify the validity of our model about the anomalous reflection, numerical simulations of the constructed metasurfaces were conducted for different distance gradients. Here, the incident angle was kept to be $0^{\circ}$. Figure 3 shows the simulated reflected fields of the designed metasurface with different unit spaces (i.e., $120 \mathrm{~mm}, 130 \mathrm{~mm}, 140 \mathrm{~mm}$, $150 \mathrm{~mm}, 160 \mathrm{~mm}, 170 \mathrm{~mm}$, and $180 \mathrm{~mm}$, respectively). The corresponding $d \phi / d x$ are $6.54 \mathrm{rad} / \mathrm{m}, 6.04 \mathrm{rad} / \mathrm{m}, 5.61 \mathrm{rad} / \mathrm{m}$, $5.23 \mathrm{rad} / \mathrm{m}, 4.91 \mathrm{rad} / \mathrm{m}, 4.62 \mathrm{rad} / \mathrm{m}$, and $4.36 \mathrm{rad} / \mathrm{m}$, separately. The reflected angles are $36^{\circ}, 34^{\circ}, 31^{\circ}, 29^{\circ}, 27^{\circ}, 25^{\circ}$, $24^{\circ}$, and $22^{\circ}$, respectively, while the theoretical reflected angles derived based on (1) should be $35.93^{\circ}, 33.17^{\circ}, 30.80^{\circ}$, $28.75^{\circ}, 26.95^{\circ}, 25.36^{\circ}, 23.96^{\circ}$, and $22.69^{\circ}$, respectively. Good agreements can be found between the simulated results and the calculated results. Although these eight units possessing strict $\pi / 4$ phase shifts are designed for the working frequency of $570 \mathrm{~Hz}$, based on [24] we can know that the shifts between adjacent units still maintain approximately $\pi / 4$ even when the frequency changes near the working frequency. Therefore, the phenomenon of abnormal reflection can still be found, but the effect of abnormal reflection may slightly decline in quality. It is worth pointing out that, except a strong reflection on the main direction, we can also find a weak reflection on the other direction. This phenomenon is caused by the phase shifts of the whole metasurface as a 1D phononic crystal [35].

\section{Applications of the Metasurface}

When acoustic waves impinge on a metasurface, the distribution of scattered pressure field follows generalized Snell's law. However, if we apply a slight modification to the metasurface, such as changing the phase gradients of different parts on it, the scattered field will be modulated to specific distribution. In the following, we show different wave manipulation effects by exploiting the proposed acoustic metasurfaces based on different combinations of fundamental units. In particular, we will show three mechanisms that could promote the development of future ultracompact acoustic devices, including an ultrathin planar acoustic axicon, an ultrathin planar lens, and a nondiffracting Airy beam $[36,37]$.

4.1. Design of a Planar Acoustic Axicon. In order to design the acoustic axicon, the reflected angle of sound wave is set to be $10^{\circ}$, which is symmetrical along $z$-axis. When the sound wave perpendicularly impinges on the surface, the phase gradient 


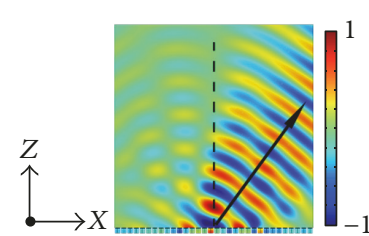

(a)

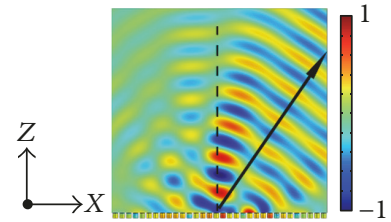

(b)

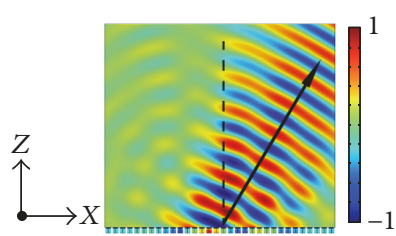

(c)

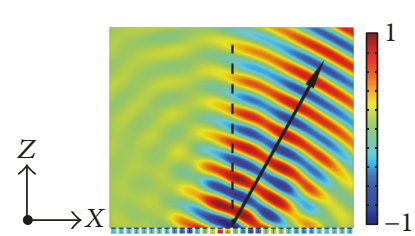

(d)

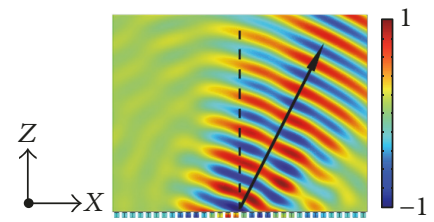

(e)

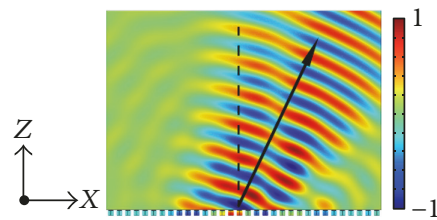

(f)

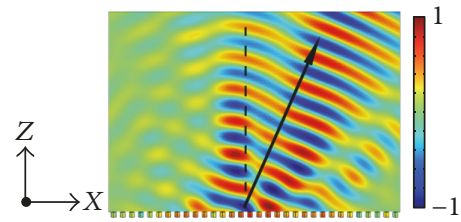

(g)

FIGURE 3: Simulated snapshots of pressure map for the reflected beams of the metasurfaces with seven different unit spaces ((a) (g) correspond to $120,130,140,150,160,170$, and $180 \mathrm{~mm}$, respectively). The metasurfaces are located on the $x$-axis. The incident waves with a Gaussian beam propagate along the negative direction of $z$-axis. The amplitudes of the acoustic pressures are normalized. The reflected angle changes with the unit space.

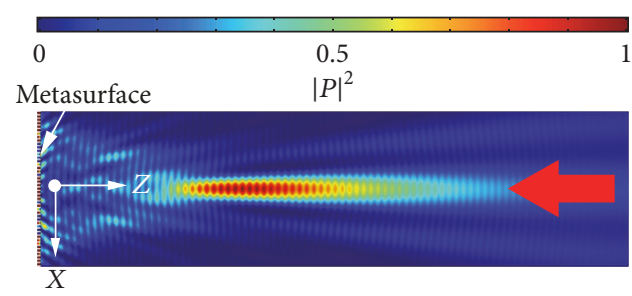

(a)

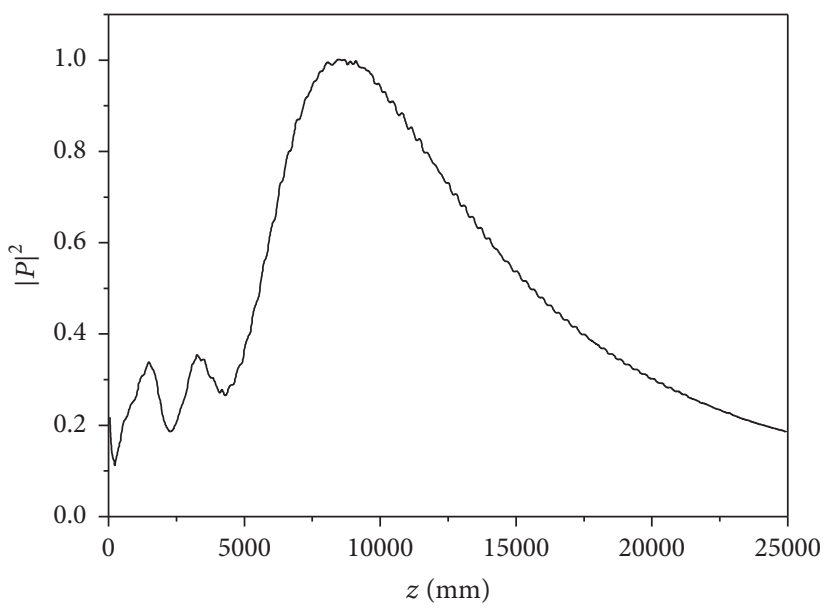

(c)

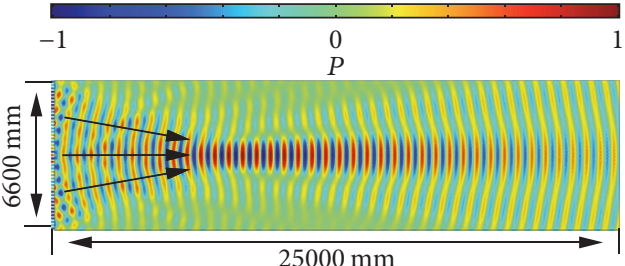

(b)

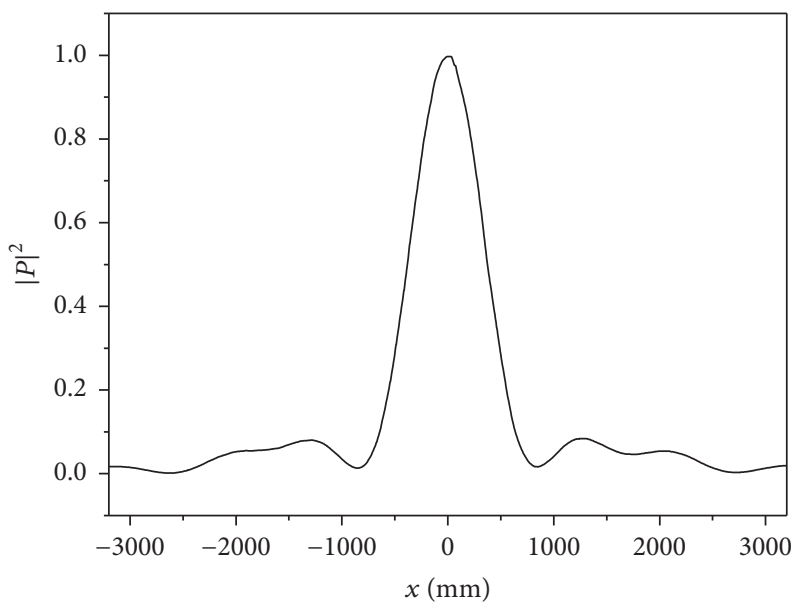

(d)

FIGURE 4: Acoustic axicon realized by the designed planar metasurface. (a) The normalized squared absolute pressure distribution of the reflected waves. The red arrow indicates the propagation direction of incident wave. (b) Transient sound pressure field distribution of reflected wave. The black arrows refer to the reflected directions. The angle between equiphase surface and $x$-axis is $10^{\circ}$. (c) The longitudinal distribution of acoustic intensity at $x=0$. (d) The transverse distribution of acoustic intensity at $z=8500 \mathrm{~mm}$.

of the metasurface along $x$-axis should satisfy the following equation:

$$
\left|\frac{d \phi}{d x}\right|=k_{0} \sin 10^{\circ}
$$

Based on (2), the planar acoustic axicon is readily constructed by symmetrically arranging the units along the positive and negative directions of $x$-axis, respectively. 43 units were employed to build this planar axicon, of which the whole size is $x \times z=6600 \times 120 \mathrm{~mm}$. The reflection behavior of this axicon was simulated using COMSOL. Figures 4(a) and 4(b) exhibit the patterns of normalized squared absolute pressure and transient pressure field of transmitted sound waves, respectively. The red arrow indicates the incident 


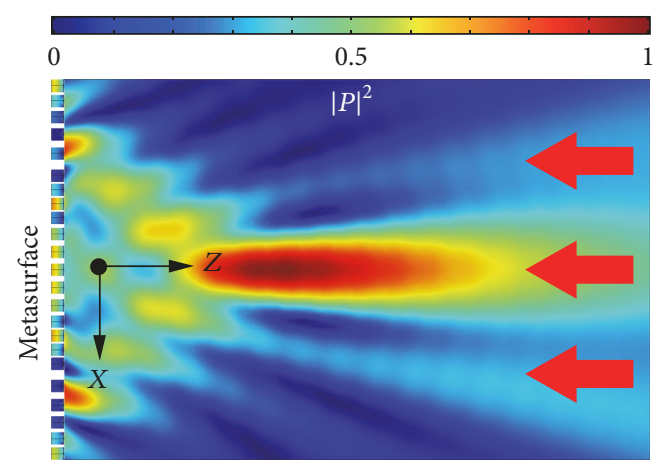

(a)

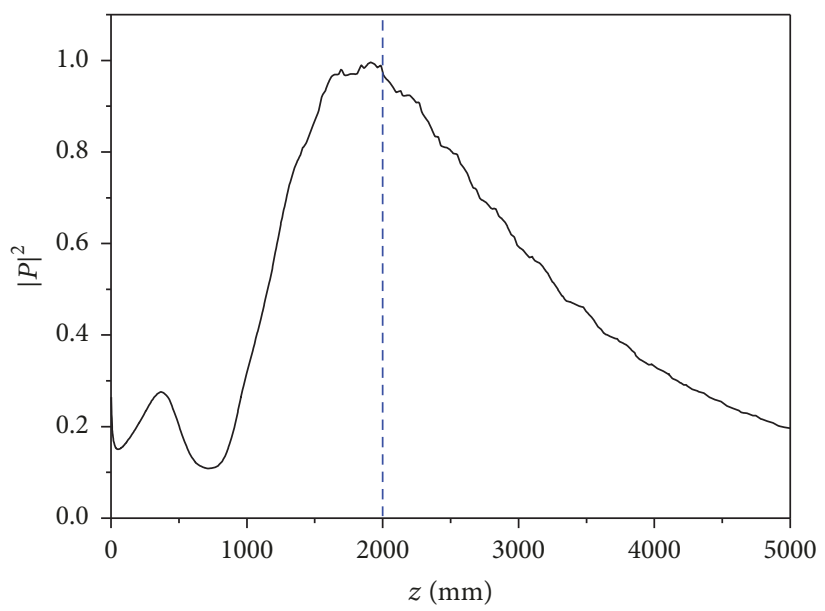

(c)

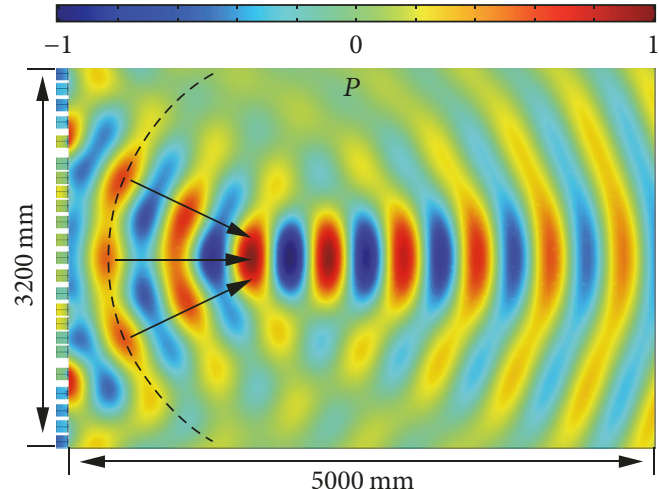

(b)

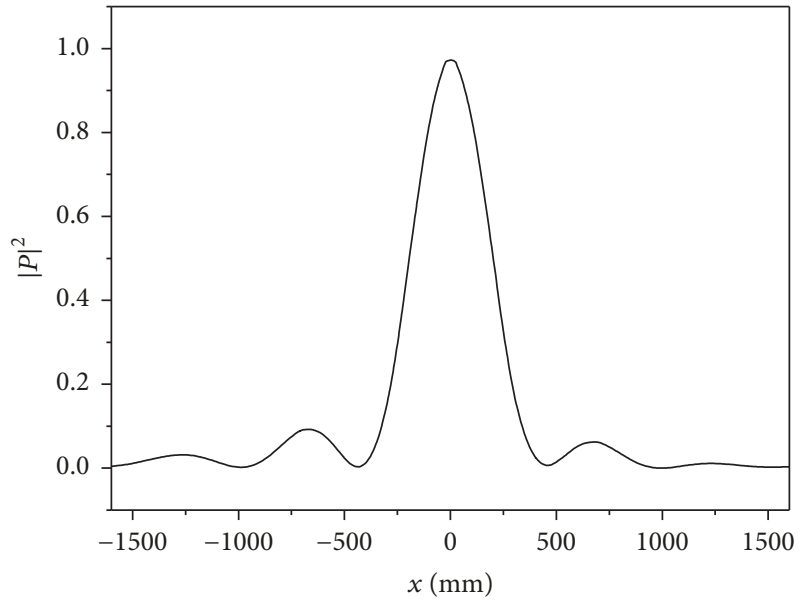

(d)

Figure 5: Planar acoustic lens. (a) The squared absolute pressure field of reflected waves. The red arrows represent the propagation direction of incident plane wave. (b) The transient pressure field distribution of reflected waves. The black dashed arc indicates the shape of reflected wavefront. (c) The longitudinal sound intensity distribution at $x=0$. (d) The transverse distribution of intensity at $z=2000 \mathrm{~mm}$.

waves. In Figure 4(a), a high-intensity focal needle can be found to form a striking contrast with ambient quiet field. It is because the reflected waves propagate along the direction marked with black arrows, as shown in Figure 4(b). As a result, the acoustic energy concentrates toward the symmetric axis of the metasurface. Figure 4(c) displays the normalized field distribution of squared absolute pressure along $z$ axis. As the coordinate increases to $8500 \mathrm{~mm}$, the acoustic intensity reaches the peak value. In addition, the focused spot is very long. For example, the acoustic intensity is over $60 \%$ of the peak within a wide coordinate region $(6000 \mathrm{~mm}<x<$ $13000 \mathrm{~mm}$ ). The field distribution of square absolute pressure along $x$-axis at $z=8500 \mathrm{~mm}$ is exhibited in Figure 4(d). The high-intensity area in transverse direction is much narrower, compared with the longitudinal field distribution.

4.2. Design of a Planar Acoustic Lens. To design a planar acoustic lens, a hyperboloidal phase profile is employed on the metasurfaces. For a given focal length $F$, the phase distribution along the surface must satisfy the following equation:

$$
\phi(x)=\frac{2 \pi}{\lambda}\left(\sqrt{x^{2}+F^{2}}-F\right)
$$

where $F$ is set to be $2000 \mathrm{~mm}$. In order to construct this planar lens, 23 units were employed. The dimension of this lens is $x \times z=3200 \times 120 \mathrm{~mm}$. The simulated squared absolute pressure of the reflected field is shown in Figure 5(a), in which a distinct focal spot appears. The red arrows indicate the incident waves. Figure 5(b) exhibits the transient pressure field distribution of reflected waves. An arc wavefront can be observed, as represented by the black dashed line. The black arrows indicate the propagation direction of reflected waves, from which we can clearly observe the phenomenon of energy convergence. The longitudinal intensity distribution at $x=0$ is shown in Figure 5(c). The peak location is exactly at the focal spot, $z=2000 \mathrm{~mm}$. Figure 5(d) shows the transverse intensity distribution at $z=2000 \mathrm{~mm}$.

4.3. Acoustic Nondiffracting Airy Beam. The acoustic nondiffracting Airy beam has unique features, such as selfbending and self-healing [38]. To realize the Airy beam using our metasurface, we need to rearrange the units according to a specific phase gradient. The bending trajectory is specified to be an $\operatorname{arc} x=f(z)=\sqrt{r^{2}-(z-r)^{2}}$ with the center at $(x, z)=(0, r)$. A plane wave propagating along the negative direction of $z$-axis is chosen as the incident beam. The desired 


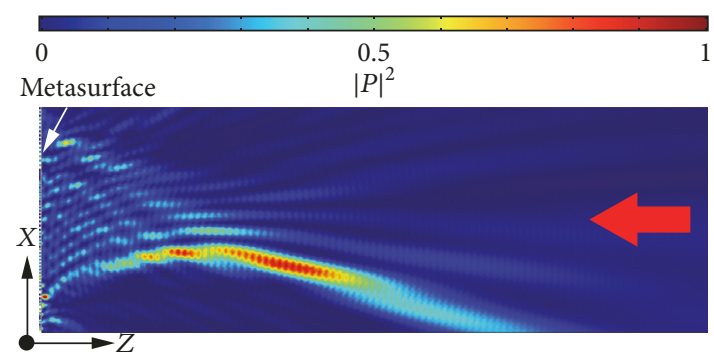

(a)

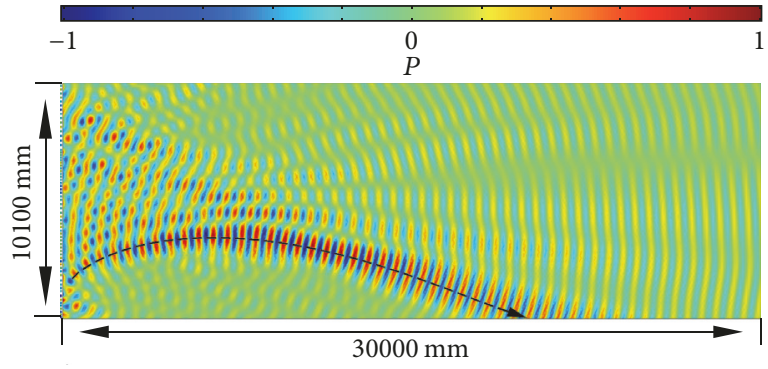

(b)

Figure 6: Acoustic nondiffracting Airy beam. (a) The squared absolute pressure field of reflected waves. The red arrows represent the propagation direction of incident plane wave. (b) The transient pressure field distribution of reflected waves. The black dashed arrow indicates the travel trajectory of the acoustic energy.

phase profile can be expressed as $\phi(x)=k_{0}(x-2 r \sqrt{x / r})$. In the simulations, the metasurface extends from $0 \mathrm{~mm}$ to $10100 \mathrm{~mm}$ along the $x$-axis. The arc radius is selected to be $r=5 \lambda$. Figures 6(a) and 6(b) exhibit the simulated squared absolute pressure and the transient pressure field distribution of the reflected waves, respectively. It is obvious that the reflected waves propagate along a bending trajectory, which makes the acoustic beam capable of bypassing obstacles on its path.

\section{Conclusion}

In summary, we designed a new kind of acoustic metasurface consisting of cubes with cylindrical cavities, whose endings are covered with elastic membrane and air gap. The simulated results demonstrate that this ultrathin acoustic metasurface (about $\lambda / 5$ ) can fully manipulate the propagation of sound waves. By employing linear phase gradient profiles, anomalous reflections were realized in a controllable manner. By utilizing nonlinear phase profiles, a planar lens and an axicon were constructed. Furthermore, an acoustic Airy beam was realized, which may find possible applications in the fields of biomedical ultrasound. As the structural dimensions of our units are the order of millimeter or even centimeter, the general 3D printing technology is ok for the future experimental preparation. The designed metasurface should open an avenue for the integration and miniaturization of acoustic equipment.

\section{Conflicts of Interest}

The author declares that there are no conflicts of interest.

\section{Acknowledgments}

This work was supported by the National Natural Science Foundation of China under Grant no. 11764033, Natural Science Foundation of Ningxia under Grant no. NZ17253, Higher School Science Research Project of Ningxia under Grant no. NGY2016173, Undergraduate Teaching Engineering Project in 2017 of Ningxia Normal University, and Ningxia Higher School First-Class Discipline Construction (Pedagogy) under Grant no. NXYLXK2017B11.

\section{References}

[1] Z. Liu, X. Zhang, Y. Mao et al., "Locally resonant sonic materials," Science, vol. 289, no. 5485, pp. 1734-1736, 2000.

[2] N. Fang, D. Xi, J. Xu et al., "Ultrasonic metamaterials with negative modulus," Nature Materials, vol. 5, no. 6, pp. 452-456, 2006.

[3] S. Yao, X. Zhou, and G. Hu, "Investigation of the negative-mass behaviors occurring below a cut-off frequency," New Journal of Physics, vol. 12, Article ID 103025, 2010.

[4] S. H. Lee, C. M. Park, Y. M. Seo, Z. G. Wang, and C. K. Kim, "Composite acoustic medium with simultaneously negative density and modulus," Physical Review Letters, vol. 104, no. 5, Article ID 054301, 2010.

[5] H. Chen, H. Zeng, C. Ding, C. Luo, and X. Zhao, "Doublenegative acoustic metamaterial based on hollow steel tube metaatom," Journal of Applied Physics, vol. 113, no. 10, Article ID 104902, 2013.

[6] S. Zhai, H. Chen, C. Ding, and X. Zhao, "Double-negative acoustic metamaterial based on meta-molecule," Journal of Physics D: Applied Physics, vol. 46, no. 47, Article ID 475105, 2013.

[7] S. L. Zhai, X. P. Zhao, S. Liu, F. L. Shen, L. L. Li, and C. R. Luo, "Inverse Doppler Effects in Broadband Acoustic Metamaterials," Scientific Reports, vol. 6, Article ID 32388, 2016.

[8] C.-L. Ding and X.-P. Zhao, "Multi-band and broadband acoustic metamaterial with resonant structures," Journal of Physics D: Applied Physics, vol. 44, no. 21, Article ID 215402, 2011.

[9] L. Fok and X. Zhang, "Negative acoustic index metamaterial," Physical Review B: Condensed Matter and Materials Physics, vol. 83, no. 21, Article ID 214304, 2011.

[10] L. Zigoneanu, B.-I. Popa, A. F. Starr, and S. A. Cummer, "Design and measurements of a broadband two-dimensional acoustic metamaterial with anisotropic effective mass density," Journal of Applied Physics, vol. 109, no. 5, Article ID 054906, 2011.

[11] Z. Yang, H. M. Dai, N. H. Chan, G. C. Ma, and P. Sheng, "Acoustic metamaterial panels for sound attenuation in the 50-1000 Hz regime," Applied Physics Letters, vol. 96, no. 4, Article ID 041906, 2010.

[12] S. A. Kuznetsov, M. A. Astafev, M. Beruete, and M. NavarroCía, "Planar holographic metasurfaces for terahertz focusing," Scientific Reports, vol. 5, article no. 7738, 2015.

[13] A. Shaltout, J. Liu, V. M. Shalaev, and A. V. Kildishev, "Optically active metasurface with non-chiral plasmonic nanoantennas," Nano Letters, vol. 14, no. 8, pp. 4426-4431, 2014. 
[14] N. Yu, P. Genevet, M. A. Kats et al., "Light propagation with phase discontinuities: Generalized laws of reflection and refraction," Science, vol. 334, no. 6054, pp. 333-337, 2011.

[15] X. Ni, Z. J. Wong, M. Mrejen, Y. Wang, and X. Zhang, "An ultrathin invisibility skin cloak for visible light," Science, vol. 349, no. 6254, pp. 1310-1314, 2015.

[16] G. Zheng, H. Mühlenbernd, M. Kenney, G. Li, T. Zentgraf, and S. Zhang, "Metasurface holograms reaching $80 \%$ efficiency," Nature Nanotechnology, vol. 10, no. 4, pp. 308-312, 2015.

[17] M. Dubois, C. Shi, Y. Wang, and X. Zhang, "A thin and conformal metasurface for illusion acoustics of rapidly changing profiles," Applied Physics Letters, vol. 110, no. 15, Article ID 151902, 2017.

[18] C. Faure, O. Richoux, S. Félix, and V. Pagneux, "Experiments on metasurface carpet cloaking for audible acoustics," Applied Physics Letters, vol. 108, no. 6, Article ID 064103, 2016.

[19] W. Tang and C. Ren, "Total transmission of airborne sound by impedance-matched ultra-thin metasurfaces," Journal of Physics D: Applied Physics, vol. 50, no. 10, Article ID 105102, 2017.

[20] B. Liu, W. Zhao, and Y. Jiang, "Apparent Negative Reflection with the Gradient Acoustic Metasurface by Integrating Supercell Periodicity into the Generalized Law of Reflection," Scientific Reports, vol. 6, Article ID 38314, 2016.

[21] X.-P. Wang, L.-L. Wan, T.-N. Chen, A.-L. Song, and F. Wang, "Broadband unidirectional acoustic cloak based on phase gradient metasurfaces with two flat acoustic lenses," Journal of Applied Physics, vol. 120, no. 1, Article ID 014902, 2016.

[22] C. Shen, Y. Xie, J. Li, S. A. Cummer, and Y. Jing, "Asymmetric acoustic transmission through near-zero-index and gradientindex metasurfaces," Applied Physics Letters, vol. 108, no. 22, Article ID 223502, 2016.

[23] C. Ding, X. Zhao, H. Chen, S. Zhai, and F. Shen, "Reflected wavefronts modulation with acoustic metasurface based on double-split hollow sphere," Applied Physics A: Materials Science \& Processing, vol. 120, no. 2, pp. 487-493, 2015.

[24] S. Zhai, H. Chen, C. Ding et al., "Ultrathin skin cloaks with metasurfaces for audible sound," Journal of Physics D: Applied Physics, vol. 49, no. 22, Article ID 225302, 2016.

[25] Y. Li, B. Liang, X.-Y. Zou, and J.-C. Cheng, "Extraordinary acoustic transmission through ultrathin acoustic metamaterials by coiling up space," Applied Physics Letters, vol. 103, no. 6, Article ID 063509, 2013.

[26] Y.-F. Zhu, X.-Y. Zou, R.-Q. Li et al., "Dispersionless manipulation of reflected acoustic wavefront by subwavelength corrugated surface," Scientific Reports, vol. 5, Article ID 10966, 2015.

[27] H. Esfahlani, H. Lissek, and J. R. Mosig, "Generation of acoustic helical wavefronts using metasurfaces," Physical Review B: Condensed Matter and Materials Physics, vol. 95, no. 2, Article ID 024312, 2017.

[28] H. Esfahlani, S. Karkar, H. Lissek, and J. R. Mosig, "Acoustic carpet cloak based on an ultrathin metasurface," Physical Review B: Condensed Matter and Materials Physics, vol. 94, no. 1, Article ID 014302, 2016.

[29] G. Ma, M. Yang, Z. Yang, and P. Sheng, "Low-frequency narrowband acoustic filter with large orifice," Applied Physics Letters, vol. 103, no. 1, Article ID 011903, 2013.

[30] C. J. Naify, C.-M. Chang, G. McKnight, and S. R. Nutt, "Scaling of membrane-type locally resonant acoustic metamaterial arrays," The Journal of the Acoustical Society of America, vol. 132, no. 4, pp. 2784-2792, 2012.
[31] C. J. Naify, C.-M. Chang, G. McKnight, and S. Nutt, "Transmission loss and dynamic response of membrane-type locally resonant acoustic metamaterials," Journal of Applied Physics, vol. 108, no. 11, Article ID 114905, 2010.

[32] A. Leblanc and A. Lavie, "Three-dimensional-printed membrane-type acoustic metamaterial for low frequency sound attenuation," The Journal of the Acoustical Society of America, vol. 141, no. 6, pp. EL538-EL542, 2017.

[33] H. Zhu and F. Semperlotti, "Anomalous Refraction of Acoustic Guided Waves in Solids with Geometrically Tapered Metasurfaces," Physical Review Letters, vol. 117, no. 3, Article ID 034302, 2016.

[34] R. Yang, D. Li, D. Gao et al., "Negative Reflecting Meta-Mirrors," Scientific Reports, vol. 7, no. 1, article 5729, 2017.

[35] K. Tang, C. Qiu, M. Ke, J. Lu, Y. Ye, and Z. Liu, "Anomalous refraction of airborne sound through ultrathin metasurfaces," Scientific Reports, vol. 4, article no. 6517, 2014.

[36] Y. Li, B. Liang, Z.-M. Gu, X.-Y. Zou, and J.-C. Cheng, "Reflected wavefront manipulation based on ultrathin planar acoustic metasurfaces," Scientific Reports, vol. 3, article no. 2546, 2013.

[37] Z. Lin, X. Guo, J. Tu, Q. Ma, J. Wu, and D. Zhang, "Acoustic nondiffracting Airy beam," Journal of Applied Physics, vol. 117, no. 10, Article ID 104503, 2015.

[38] P. Zhang, T. Li, J. Zhu et al., "Generation of acoustic self-bending and bottle beams by phase engineering," Nature Communications, vol. 5, article no. 4316, 2014. 

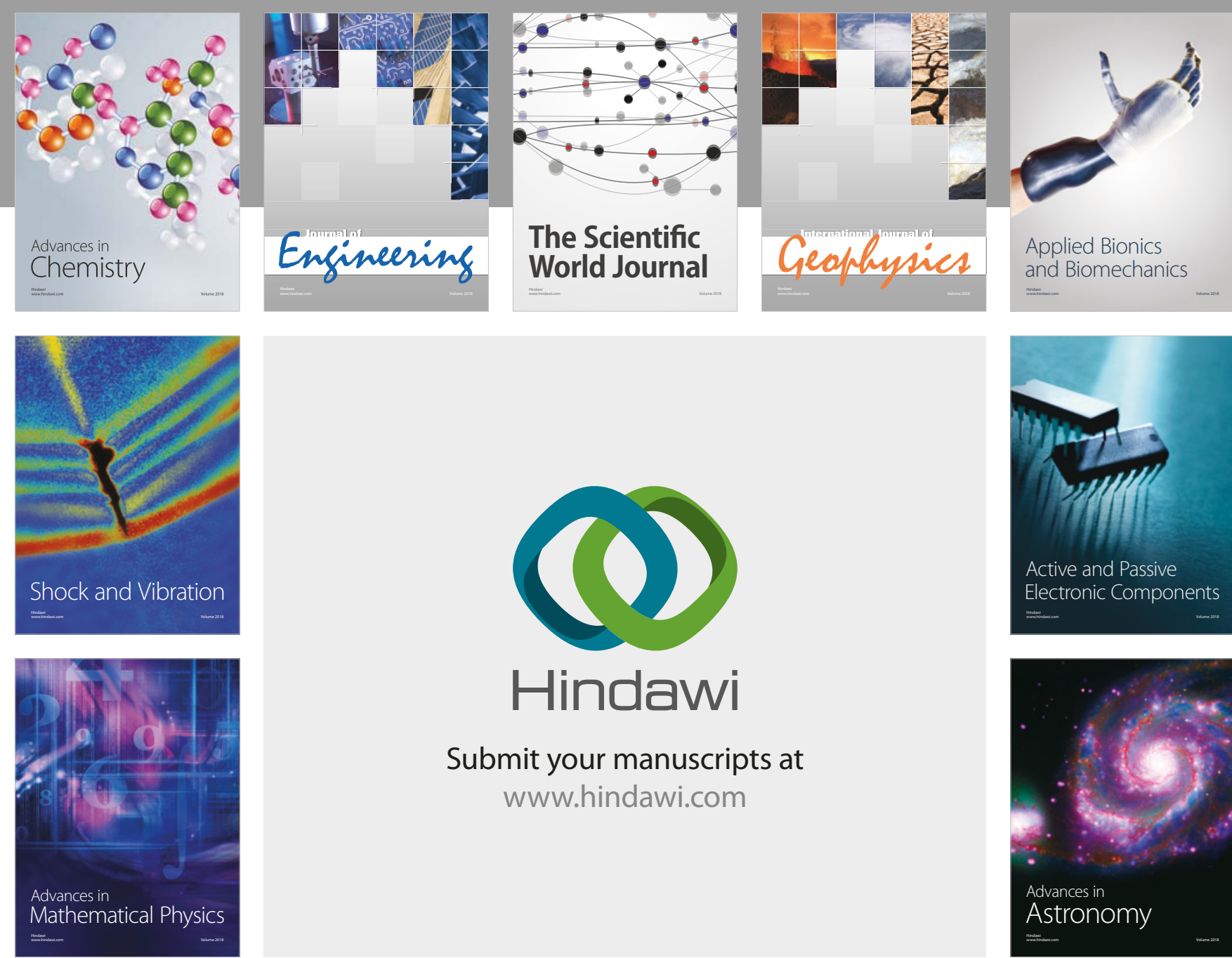

Submit your manuscripts at

www.hindawi.com

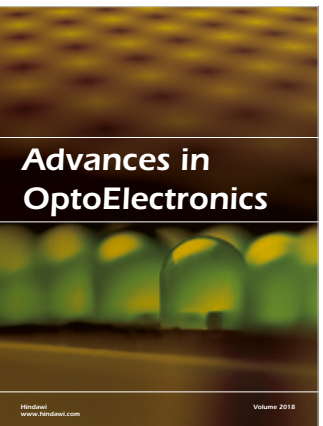

\section{Rotcting Machinery}
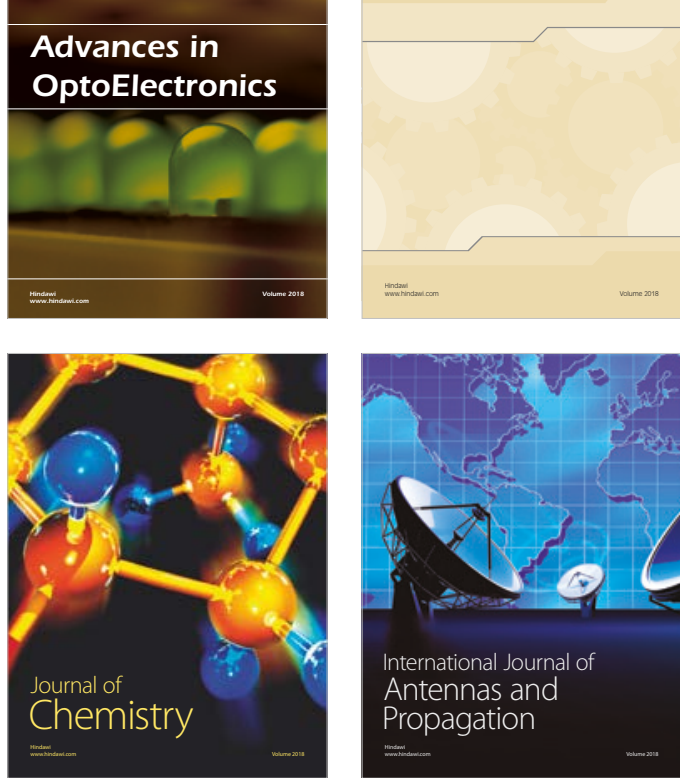

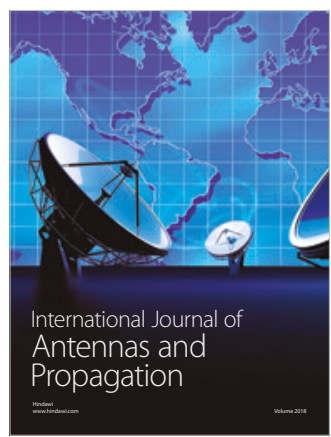

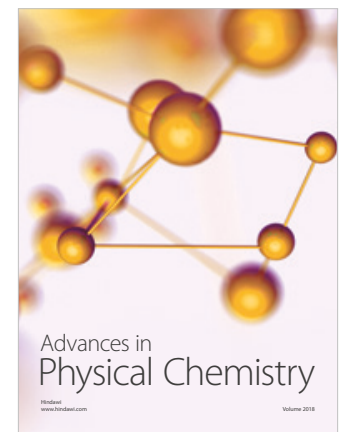

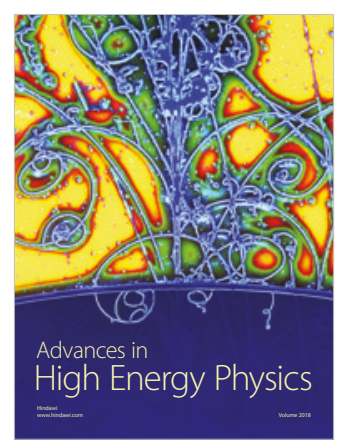

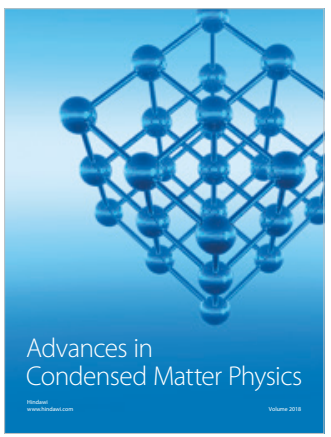

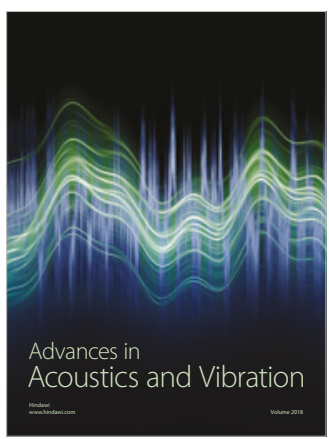

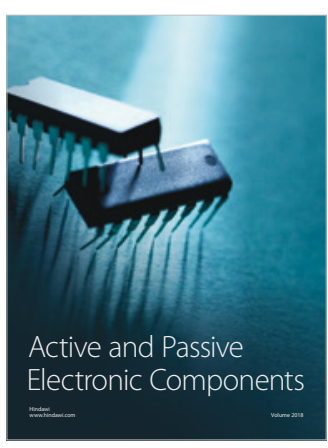
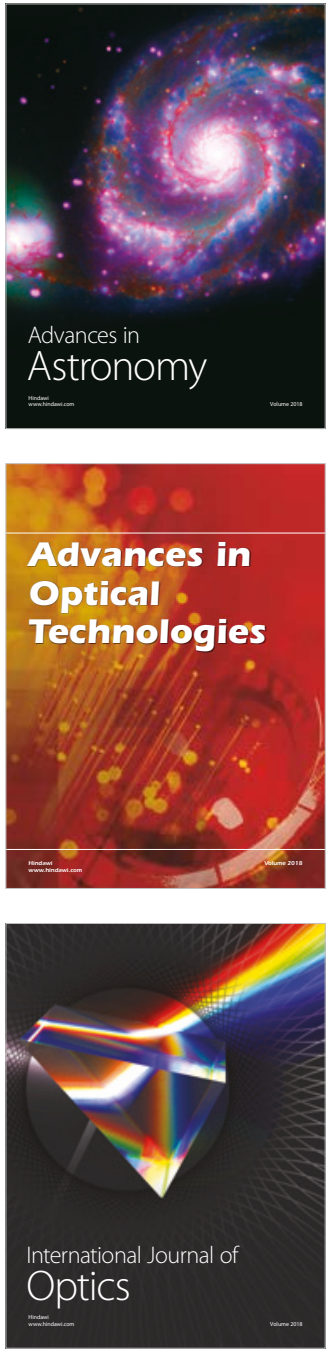